\title{
Aplicación de un instrumento de estimación de capacidades funcionales en una trabajadora con obesidad mórbida
}

\author{
Application for a functional capacity estimation instrument on a worker with \\ morbid obesity
} Caridad León Robles', Esperanza Álvarez Maeztu', Mercedes Petralanda Aburto', Isabel Larruscain Garay',
Izaskun Sáez Larrucea'

1. Servicio de Prevención de Riesgos Laborales (SPRL). Unidad Básica de Prevención. Hospital Universitario de Cruces. Bizkaia. España.

Recibido: 23-04-2018

Aceptado: 28-02-2019

\section{Correspondencia}

Caridad León Robles

Servicio de Prevención de Riesgos Laborales (SPRL).

Unidad Básica de Prevención. Hospital Universitario de Cruces.

Plaza de Cruces, s/n.

48903 Baracaldo. Bizkaia. España.

Correo electrónico: caridadpilar@gmail.com

Resumen

Introducción: Las recomendaciones sobre la valoración de aptitud de trabajadores con riesgos de exposición de carga física mencionan que en condición de especial sensibilidad no sería aplicable el criterio de guías técnicas, por lo que habría que realizar una evaluación específica y un análisis de perfil del puesto, ajustando los resultados a las capacidades funcionales del trabajador. En una intención de facilitar a los clínicos esta orientación, hemos propuesto el uso del formulario de capacidades funcionales de la Secretaría del Consejo de la Tesorería de Canadá.

Objetivo: Aplicar un instrumento de uso sencillo para estimar la capacidad funcional de una trabajadora con obesidad grado III para un puesto de Auxiliar de Enfermería en el Servicio de Esterilización de un Hospital Terciario.

Materiales y métodos: Se realizó un examen médico completo, el análisis del puesto de trabajo y se completó el formulario en mención. El conjunto de datos obtenidos se confrontan para la identificación de desajustes y riesgos y la toma de decisiones final.

Resultados: Se encontraron importantes desajustes entre las condiciones de la trabajadora y las características del puesto de trabajo, así como un considerable aumento del riesgo de trastornos músculoesqueléticos, por lo que se decidió declarar a la trabajadora NO APTA para el puesto de Auxiliar de Enfermería en el Servicio de Esterilización en el momento actual. Acto seguido se le oferta el puesto de consultas de Pre

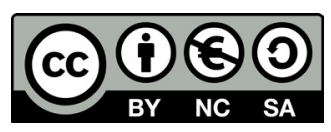

https://creativecommons.org/licenses/by-nc-sa/4.0/

Aplicación de un instrumento de estimación de capacidades funcionales en una trabajadora con obesidad mórbida Caridad León Robles, Esperanza Álvarez Maeztu, Mercedes Petralanda Aburto, Isabel Larruscain Garay, Izaskun Sáez Larrucea 
Anestesia, ya que no se encontraron desajustes en la aplicación del formulario para este puesto. Tras una reevaluación al mes de la trabajadora está satisfecha y la supervisora del servicio nos transmite que desempeña su trabajo adecuadamente.

Conclusiones: El Formulario Canadiense de Estimación de Capacidades Funcionales, traducido y modificado es una herramienta útil para orientarnos en la valoración funcional de un personal sensible para un puesto concreto, así como en la propuesta de adaptación a un puesto compatible.

Med Segur Trab (Internet). 2019;65(255):160-72

Palabras clave: Obesidad, capacidades funcionales, manejo de carga.

Abstract

Introduction: The recommendations on the aptitude assessment of workers with cargo-handling exposure risks mentions that under special sensitivity condition the criterion of technical guide would not be applicable. Therefore, a specific evaluation and profile analysis of the position should be carried out, adjusting the results to the functional capacities of the worker. In order to guide clinicians, we have proposed the use of the Functional Capabilities Form (FCF) of the Treasury Council Secretariat of Canada.

Goal: To apply a tool in order to estimate the functional capacity of a worker with Grade 3 overweight for a nursing assistant job in the sterilization service of a Tertiary Hospital.

Material and Methods: A complete medical examination was carried out, and the analysis of the work position and the aforementioned form was as well completed. The body of data obtained is confronted for the identification of imbalances and risks, and for the final decision.

Results: Significant imbalances were found between the conditions of the worker and the characteristics of the job, as well as a considerable increase in the risk of musculoskeletal disorders. So it was decided to declare the worker unsuitable for the position of nursing assistant in the sterilization service at the present time. Straight away, the pre Anesthesia consultation position was offered, since no negatives were found in the application of the Form in this position. After a re-evaluation a month later, the worker is satisfied and the supervisor of the service tells us that she is performing her job properly.

Conclusion: The Canadian Form for Functional Capabilities Estimation, translated and modified, is an useful tool to guide us on the functional assessment of a sensitive staff for a specific job, as well as on the adaptation proposal to a compatible position.

Med Segur Trab (Internet). 2019;65(255):160-72

Keywords: Obesity, Functional abilities, Cargo handling. 


\section{ANTECEDENTES}

En el marco de las actividades de vigilancia de la Salud se nos presenta el caso de una trabajadora con Obesidad Mórbida a la que tenemos que realizarle un reconocimiento médico inicial para el puesto de Auxiliar de Enfermería en el servicio de Esterilización. La tarea más habitual en este servicio, entre otras, es la manipulación de cargas. Las recomendaciones sobre la valoración de aptitud de trabajadores con riesgos de exposición de carga física indican que en aquellos casos en que haya que valorar la aptitud en un trabajador que presente unas características de peso, talla o musculatura especiales, gigantismos, enanismos, obesidad o miopatías y, que por su especial sensibilidad, no sea aplicable el criterio de la guía técnica del INSHT, será necesaria realizar una evaluación específica y un análisis de perfil de puesto de trabajo, ajustando su resultado a las capacidades funcionales del trabajador ${ }^{1}$. Es así que buscamos estimar la capacidad funcional de dicha trabajadora, dada su condición de personal sensible, aplicando un instrumento de uso sencillo que nos sirviera de apoyo en el análisis de confrontación con las exigencias del puesto en mención.

\section{DESCRIPCIÓN DEL CASO}

La trabajadora es una Auxiliar de enfermería de 39 años sin experiencia laboral en el sector sanitario, que acude al reconocimiento médico inicial para un contrato de sustitución en el Servicio de Esterilización de un Hospital Terciario.

Antecedentes Laborales: Ha trabajado de Auxiliar Administrativo y como encuestadora por teléfono y presencial durante 11 años. No ha tenido enfermedades profesionales, ni accidentes de trabajo y no tenía discapacidades reconocidas.

Antecedentes Patológicos: La trabajadora esta diagnosticada de Hipertensión arterial, cuadro ansioso que requirió tratamiento farmacológico en la juventud y que se mantiene hasta la fecha. Desorden de alimentación con episodios de atracones que se asoció al incremento de peso al iniciar estudios universitarios desde el año 2006, obesidad Grado III según clasificación de la OMS, valorada y tratada en endocrinología llegando a perder hasta $30 \mathrm{~kg}$ con posterior recuperación en los siguientes años. Por último, disnea grado II de la mMRC (modified Medical Research Council).

Examen Físico: Talla: $152 \mathrm{~cm}$, Peso: $143 \mathrm{~kg}$, IMC: $62 \mathrm{~kg} / \mathrm{m}^{2}$, diámetro abdominal: $144 \mathrm{~cm}$; TA: 112/51, FC 79/min.

Exploración Osteomuscular: Aceptable balance articular en 4 extremidades, ROT ++ , FM: 4/5. Flexión de columna a $18 \mathrm{~cm}$ del suelo, extensión limitada. Actitud de valgo en ambas rodillas, pies planos y ampliación de la base de sustentación a la marcha con separación de talones $8-10 \mathrm{~cm}$. Oximetría de pulso indica 94\% tras exploración.

Estudios Complementarios: Radiografía de tórax, EKG y Analítica general sin alteraciones. Gasometría: PH 7.41 PO2 73mg PCO2 39. Saturación de oxigeno 95\%. Espirometría y Volúmenes Pulmonares: FVC 2.96 (91.1\%); FEV1 2.45 (94\%) FEV1/FVC 83; Flujo espiratorio pico 3.831 (64.1\%).

\section{Otros Estudios Complementarios}

- Cuestionario de Somnolencia de Epworth: 7 Puntos: Normal.

- Escala de Marcha-Equilibrio de Tinetti: 19 Puntos: Riesgo de caídas. 


\section{DATOS SOBRE EL ÁREA/CONDICIONES DE TRABAJO}

\section{Servicio de Esterilización}

La central de Esterilización es una dependencia situada en el Sótano 2 del hospital, que ocupa una superficie útil de 164,7 $\mathrm{m}^{2}$ y que consta de 4 zonas: (I) Zona de recepción de material, que es la más extensa, (II) zona de preparación y sellado que además contiene el Esterilizador plasma Gas $\left(\mathrm{H}_{2} \mathrm{O}_{2}\right)$, (III) la zona de esterilización de Óxido de Etileno con dos aireadores y (IV) la zona de Autoclaves y la zona de limpio.

La actividad consiste en la recepción del material proveniente de las distintas zonas del hospital para su esterilización, clasificación y posterior distribución por el celador correspondiente. El servicio cuenta con un total de 12 trabajadoras (Auxiliares de Enfermería y una supervisora Enfermera) distribuidas por turnos (4 de mañana, 6 de tarde y 2 en la noche).

\section{Puesto de Auxiliar de Enfermería}

\section{Tareas:}

- Recepción, clasificación y distribución del material a esterilizar según proceso. La mayoría de estos materiales proceden de los quirófanos y área de urgencias.

- Introducción del material en los diferentes equipos de esterilización.

- Control de los diferentes procesos de Esterilización.

- Descarga y colocación del material esterilizado en carros de distribución.

\section{Demandas:}

- Movilización manual de cargas multitarea con pesos entre 3-14 kg a diferentes niveles y relación a ciclos de los equipos de estilización (Autoclave y Plasma 15 a 10 ciclos turno).

- Bipedestación prolongada el $75 \%$ de la Jornada y el resto del tiempo en sedestación (al usar la selladora) o caminando.

- Transporte y arrastre de carros y armarios en espacios reducidos (Fig. 1, Fig. 2).
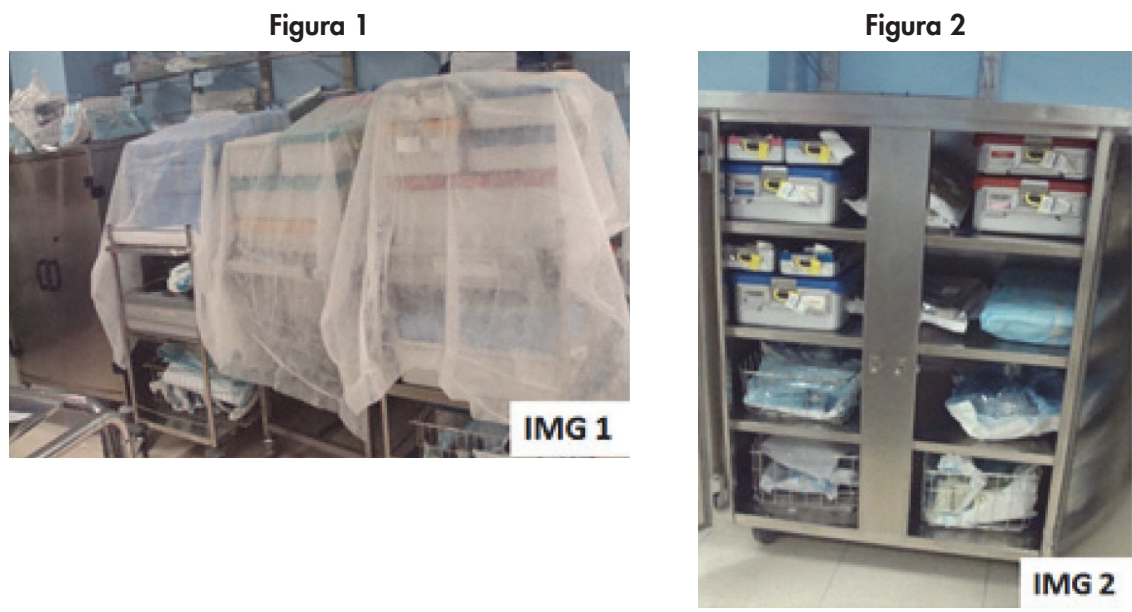

\section{Entorno y dimensiones:}

- Los mandos de algunos equipos se encuentran a $190 \mathrm{~cm}-200 \mathrm{~cm}$ del suelo (Fig. 3).

- Existe dificultad de desplazamiento al acumularse carros y armarios con material generándose pasillos estrechos y zonas de paso obstaculizadas (Fig. 1).

- La temperatura ambiente puede variar desde $21^{\circ}$ a $25.8^{\circ} \mathrm{C}$. 
Figura 3

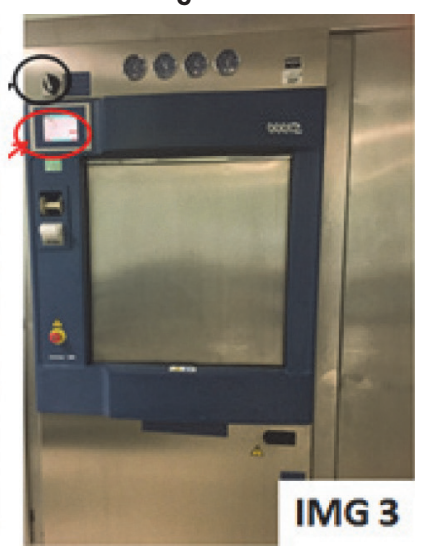

\section{CRITERIOS DE REFERENCIA/DOCUMENTACIÓN APLICABLE}

La base jurídica se encuentra en la vigente Ley de Prevención de Riesgos Laborales que su art. 25, Capítulo III $^{2}$, expone que el empresario garantizará de manera específica la protección de los trabajadores que, por sus propias características personales o estado biológico conocido, incluidos aquellos que tengan reconocida la situación de discapacidad física, psíquica o sensorial, sean especialmente sensibles a los riesgos derivados del trabajo.

\section{MATERIAL Y MÉTODO}

Para determinar la capacidad funcional de esta trabajadora, realizamos un examen médico exhaustivo y a su vez un análisis específico del puesto de trabajo en coordinación con el departamento Técnico del servicio de Prevención. Ante la dificultad de la aplicabilidad de la Guía Técnica para la evaluación y prevención de los riesgos relativos a la manipulación manual de cargas del Instituto Nacional de Seguridad Salud e Higiene en el Trabajo (INSHT) ${ }^{3}$, se realizó una búsqueda bibliográfica de un instrumento de uso rápido y sencillo para el apoyo en la toma de decisiones donde seleccionamos el Formulario de Capacidades Funcionales de la Secretaría del Consejo de la Tesorería de Canadá, el cual está descrito como un instrumento que refleja la mejor práctica de los profesionales de la salud para obtener información en caso de enfermedad o de lesión de un trabajador, permitiendo tomar las medidas de adaptación de un trabajador enfermo, o lesionado, a fin de que permanezca en el trabajo o si su ausencia es inevitable, que vuelva al trabajo tan pronto como sea posible ${ }^{4}$. Este instrumento consta de tres secciones:

Sección A: Datos del trabajador y lugar de trabajo.

Sección B: Datos de la capacidad funcional requerida para el puesto que se cumplimenta con los datos proporcionados por el supervisor del servicio y consta de varios apartados cada uno con diversas especificaciones:

- B1- Movimientos de la Columna Vertebral

- B2- Actividades en Posición sentada

- B3- Actividades en Posición de pie

- B4- Levantar/Transportar/Empujar/Tirar (Descritas en Kg)

- B5- Trabajo con Hombros/ Codos/Muñecas/manos/dedos

- B6- Actividades que requieran usos de los sentidos

- B7- Ambiente Físico de Trabajo (Sobre sus características y riesgos potenciales)

Aplicación de un instrumento de estimación de capacidades funcionales en una trabajadora con obesidad mórbida Caridad León Robles, Esperanza Álvarez Maeztu, Mercedes Petralanda Aburto, Isabel Larruscain Garay, lzaskun Sáez Larrucea 
- B8- Capacidades no Físicas relacionadas con el Trabajo (Emocional, social y cognitivo mental y de ajustarse a horarios y normas establecidas)

Sección C: Es cumplimentada por el Médico de Trabajo que interpreta y concluye sobre las posibles restricciones y/o limitaciones encontradas para desarrollar dicho puesto.

Se procedió a traducirlo al castellano y le denominamos "Formulario de Estimación de Capacidades Funcionales de la Tesorería de Canadá, modificado y traducido al Español” (FECFmte). La traducción se realizó a partir de las versiones en inglés y francés procurando mantener en todo momento el lenguaje cotidiano y de fácil comprensión que mantiene la versión original. La sección B del FECFmte se fue cumplimentado con la información proporcionada por la supervisora del Servicio de Esterilización y, además, en consulta lo complementamos con los datos del análisis del puesto y del examen médico, con la finalidad de identificar posibles desajustes y riesgos que se describieron en las conclusiones del "Formulario de Estimación de Capacidades Funcionales de la Tesorería de Canadá, modificado y traducido al Español" para clarificar la toma de decisiones sobre la aptitud de la trabajadora.

\section{RESULTADOS}

\section{Desajustes y Riesgos encontrados}

El puesto de Auxiliar de Enfermería del servicio de Esterilización presenta una demanda importante de manipulación manual de cargas de peso variable y a diferentes niveles, lo que, al confrontarlo con la constitución de la trabajadora, nos hace pensar que aumentaría considerablemente el riesgo de Trastornos Musculo-Esqueléticos (TME). Es así que el uso del FECFmte (Fig. 4, Fig. 5) nos facilitó visualizar los posibles desajustes entre las tareas del puesto y la capacidad funcional de la trabajadora. Tras su aplicación, identificamos desajustes que nos permitieron esquematizar las tareas del puesto de forma sencilla para la toma de decisiones.

La sección B del FECFmte, nos permitió esquematizar las tareas en conflicto. Posteriormente describimos en la sección C las limitaciones identificadas en cinco apartados de la sección $\mathrm{B}$.

Apartado B-1 sobre los movimientos de la columna vertebral, las tareas requerían inclinaciones de la columna, así como rotación y torsión del tronco para disponer de los materiales ubicados en los carros e introducirlos y sacarlos de los equipos de esterilización.

Apartado B-3 de las actividades en posición de pie, el 75\% de la Jornada laboral se desarrollaba en bipedestación dinámica y transitar distancias de hasta 5 metros.

Apartado B-4 sobre Levantar/Transportar/empujar/tirar en este servicio las tareas requieren levantar cargas por encima del hombro y coger cargas ubicadas en baldas que se encuentran por debajo de la rodilla, por lo tanto hay que agacharse e incluso ponerse de cuclillas varias veces por turno para llegar a las baldas inferiores de los carros de esterilización, así como empujar y arrastrar carros con material quirúrgico, que en varios casos pueden pesar hasta 25 kilos.

Apartado B-5 destaca la necesidad del uso de extremidades superiores por encima del hombro hasta por debajo del hombro y la manipulación de objetos que requieran agarre fuerte. Esto incluye la manipulación de botones del autoclave que se encuentran aproximadamente a dos metros del suelo y que se necesita mantenerlo pulsado por un minuto para iniciar el proceso.

Por último en el Apartado B-7, sobre el Ambiente Físico del Trabajo, resaltamos que es una dependencia cerrada donde el ruido y distracciones están presentes, así como condiciones de temperaturas ocasionadas por el calor del área y los vapores propios de las tareas. En este punto se hace mención sobre los riesgos potenciales en ambiente de trabajo donde identificamos como riesgos a la caída de objetos, ruido continuo, postura forzada, movimientos repetitivos, bipedestación prolongada y manejo de equipo pesado.

Aplicación de un instrumento de estimación de capacidades funcionales en una trabajadora con obesidad mórbida Caridad León Robles, Esperanza Álvarez Maeztu, Mercedes Petralanda Aburto, Isabel Larruscain Garay, Izaskun Sáez Larrucea 
En la sección C, complementamos los datos con información sobre tratamiento y diagnósticos de la trabajadora.

Figura 4

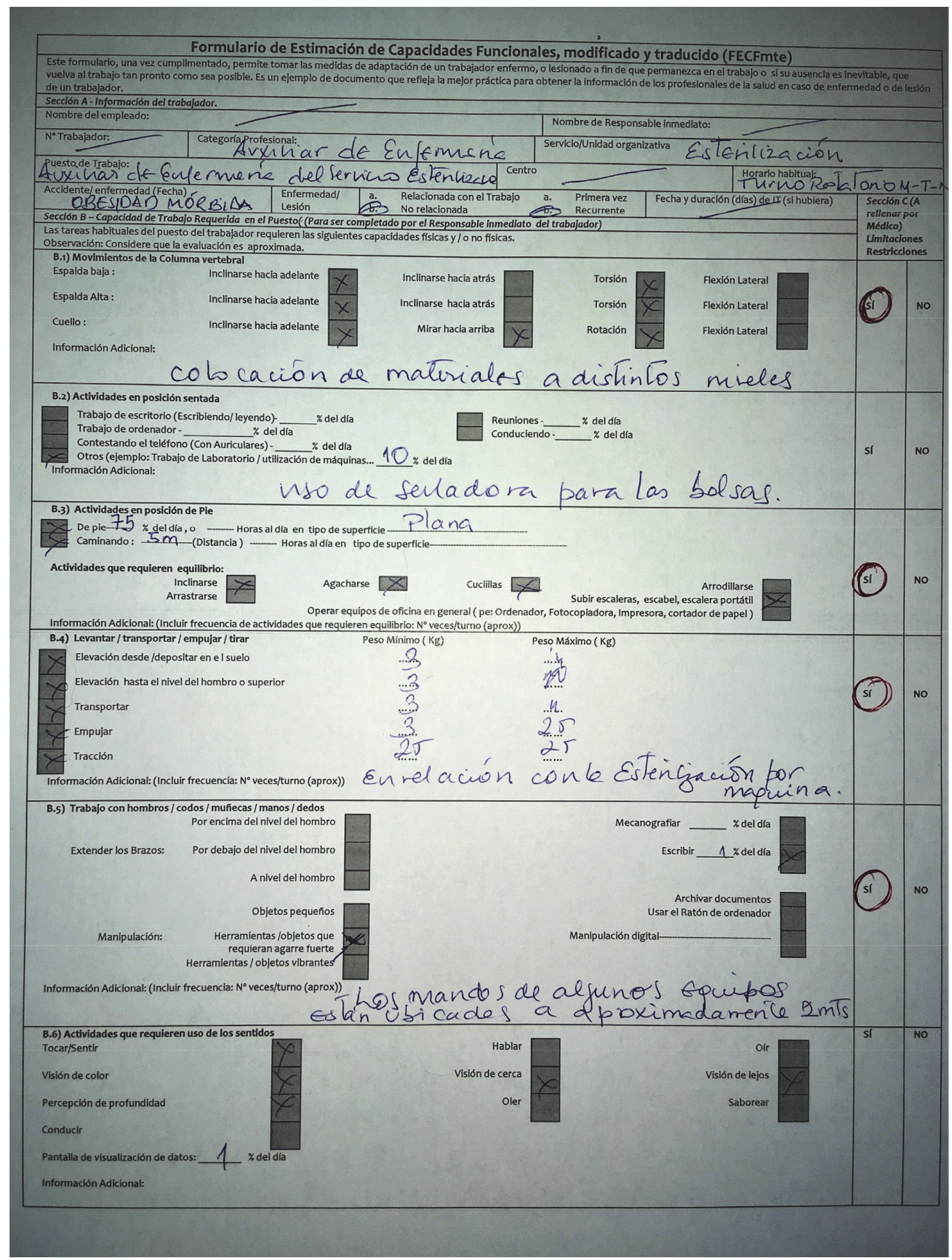

Aplicación de un instrumento de estimación de capacidades funcionales en una trabajadora con obesidad mórbida Caridad León Robles, Esperanza Álvarez Maeztu, Mercedes Petralanda Aburto, Isabel Larruscain Garay, Izaskun Sáez Larrucea 


\section{Figura 5}

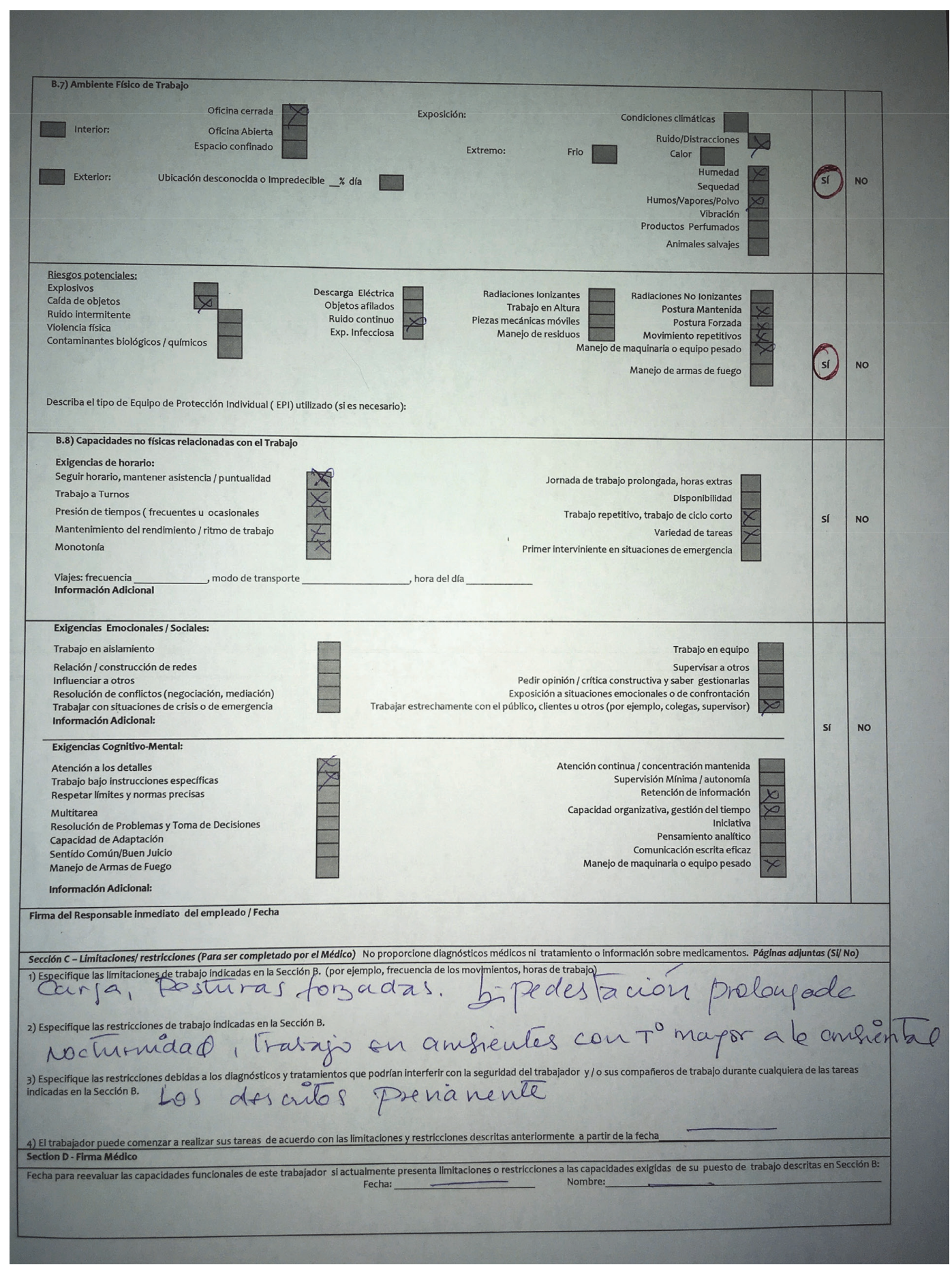

\section{Toma de decisiones}

Frente a los importantes desajustes entre las características de la trabajadora y las características del puesto de trabajo y principalmente el considerable aumento del riesgo de trastornos musculo esqueléticos (TME).

Se considera a la trabajadora NO APTA para el puesto de Auxiliar de Enfermería en el Servicio de Esterilización en el momento actual.

Acto seguido y tras viabilizar las consultas de especialidad para tratar la Obesidad, se le hace una valoración para el puesto de "Consultas de Pre Anestesia - Hospital de 
día" cuyas características son: tres consultas, dos trabajadores (una Enfermera y una Auxiliar) con una media de 15 a 20 pacientes por turno, donde las principales tareas son: atender las llamadas telefónicas, organizar las historias clínicas, recepción de pacientes, toma de constantes habituales, peso y talla, cerrar las consultas y archivar las historias en caso que sea necesario para su escaneo.

Tras la aplicación del FECFmte no se encontraron desajustes para el desempeño de este puesto en mención. Por lo que se le consideró APTA para este puesto.

Posteriormente se realizó una reevaluación pasados el primer y segundo mes de contrato de Auxiliar de Enfermería en Consultas de Pre Anestesia del Hospital de día. Nos encontramos con una trabajadora satisfecha en el puesto actual y la supervisora del servicio nos transmitió que la trabajadora realiza su trabajo adecuadamente y sobre la media.

\section{DISCUSIÓN}

La obesidad per se representa un factor de riesgo para el inicio de problemas musculo-esqueléticos $5,6,7,8,9,10,11,12$ y este riesgo se duplica si estamos frente a una obesidad mórbida ${ }^{5}$. Las zonas más frecuentemente afectadas son la columna, muñecas, hombros, caderas, rodillas y tobillos. Los diagnósticos más conocidos son los relacionados con la osteoartritis de rodilla (con un OR de 14,7), síndrome del túnel del carpo (con un OR de $4^{5}$ ), tendinitis del manguito rotador o síndrome de choque de hombro, tendinitis de Aquiles, fascitis plantar, disfunción del tendón tibial posterior acompañado o no de deformidad de pie plano, fracturas por estrés y pies de Charcot entre otros ${ }^{6}$.

En el caso de nuestra trabajadora, la importante demanda de carga física del puesto de Auxiliar de Enfermería en el Servicio de Esterilización, le obligaría a una mecánica anormal sobre sus movimientos corporales debido a su peso, aumentando así el riesgo de TME. El IMC afecta a la cinemática del tronco durante el levantamiento de cargas tanto en el plano transversal como en el plano sagital, ya que durante la flexión anterior del tronco la región lumbar sufre los mayores daños y por lo tanto mayor posibilidad de $\mathrm{TME}^{5,7}$. Las actividades que se llevan a cabo en bipedestación prolongada aumentan la probabilidad de artrosis de cadera y rodillas ${ }^{7,8}$. La presencia de patología osteomuscular llevará a la consiguiente posibilidad de absentismo laboral ${ }^{13}$. Este aspecto se ha detectado en trabajadores obesos, cuyo riesgo es de dos a tres veces mayor de terminar en una discapacidad, en comparación con sujetos de peso normal, siendo la causa principal los TME $^{5}$.

La fuerza muscular disminuida en el caso de las personas obesas se ha relacionado a la sarcopenia y a la menor capacidad oxidativa del músculo en el obeso, siendo de esta manera un músculo menos eficiente ${ }^{3}$. Los cambios en la masa y composición corporal que afectan negativamente a la fuerza muscular derivan entre 6 a $10 \%$ menor fuerza que los sujetos con peso normal ${ }^{5}$, condición que en la exploración de la trabajadora es evidente en las 4 extremidades.

Así mismo, la disminución de la capacidad ventilatoria promovida por la elevación del diafragma y la baja flexibilidad de la caja torácica que condicionan cambios en la mecánica respiratoria tanto en posición supina como en sedestación y contribuyen a modificar los valores de los volúmenes pulmonares, dificulta tareas cotidianas como caminar, subir, bajar escaleras, ponerse en cuclillas, alcanzar objetos ocasionando un notable gasto energético ${ }^{5}$. Lo que podría explicar la disnea basal tipo II y la saturación en reposo del 95\%, así como la leve desaturación tras el esfuerzo físico mientras se realizaba la exploración osteomuscular en esta trabajadora. No se observó Hipercapnea, ni alteraciones significativas instaladas en los volúmenes pulmonares como suelen verse en la mayoría de obesos ${ }^{13,14}$.

Aplicación de un instrumento de estimación de capacidades funcionales en una trabajadora con obesidad mórbida Caridad León Robles, Esperanza Álvarez Maeztu, Mercedes Petralanda Aburto, Isabel Larruscain Garay, lzaskun Sáez Larrucea 
Pese a que la exploración osteomuscular de la trabajadora mostraba un balance articular aceptable, en aspectos del FECFmte secciones B1, B3 y B4, B5 sobre la capacidad funcional requerida en los movimientos de la columna vertebral, bipedestación prolongada y trabajo con las extremidades superiores, encontramos importantes desajustes que se sustentan en las limitaciones funcionales en el aparato locomotor relacionadas a la obesidad que se explican por el aumento de rigidez en la porción dorsal de la columna vertebral, la disminución del rango de movimiento en el codo y la muñeca y el intento de compensación que provoca una baja tolerancia a posturas forzadas. También encontramos ralentización en la velocidad de los movimientos de las manos y codo ocasionando que tengan problemas para desarrollar diversas tareas durante la jornada laboral ya que llegan a ser menos precisos especialmente en aquellas que requieren movimientos de las extremidades superiores mientras está de pie ${ }^{5,6}$.

En la sección B7 sobre Ambiente Físico de Trabajo observamos de forma más clara el riesgo de accidentabilidad ${ }^{3}$ teniendo en cuenta la generación de pasillos angostos debido al material esterilizado durante la jornada laboral. En los trabajadores obesos las tareas realizadas con los miembros superiores, mientras se encuentra en bipedestación, parecen estar vinculadas a un mayor riesgo de caídas y, por consiguiente, a un aumento de la posibilidad de accidentabilidad 5 . El patrón motor al caminar se caracteriza por una reducción en la longitud, reducción de la frecuencia del paso, ampliación de la base de sustentación y rotación externa de los pies, posiblemente como una reorganización neuromuscular para no sobrecargar las rodillas. El centro de gravedad se desplaza hacia adelante ya que la lordosis lumbar aumenta junto con la cifosis dorsal y la lordosis cervical, por lo que en la bipedestación tienen una actitud de valgo en las rodillas y con frecuencia coexisten pies planos en un intento compensatorio de modificar el centro de gravedad $^{5,8,10,11}$. Por lo que los sujetos obesos encuentran dificultades interactuando con el espacio circundante, muebles, herramientas, ropa y tareas que son básicamente diseñadas para sujetos de peso normal. Sin embargo, en cuanto a las lesiones traumáticas del trabajo, pocos estudios informan que el riesgo es mayor en sujetos obsesos y solo un estudio concluye que la reducción de peso podría prevenir lesiones traumáticas ${ }^{5,15}$.

Por último, en la sección C tenemos en cuenta el diagnóstico de la obesidad e hipertensión de la trabajadora. Situación que toma relevancia según los descrito en el informe de ANSES (Agence nationale de sécurité sanitaire de l'alimentation, de l'environnement et du travail) del $2016^{16}$, sobre Nocturnidad y riesgos en el trabajador, ya que menciona una asociación entre la nocturnidad y el síndrome metabólico con sus consiguientes alteraciones metabólicas a largo plazo, dificultando más el control del peso y aumentando el riesgo cardiovascular. Por otro lado, la Trabajadora presenta una disnea grado II que, unida a la fuerza muscular disminuida, dificultarán el desarrollo de trabajos que requieran esfuerzo físico constante en un ambiente a temperaturas mayores a las recomendadas para incluso para trabajos ligeros ${ }^{17}$.

\section{CONCLUSIONES}

El "Formulario de Estimación de Capacidades Funcionales de la Tesorería de Canadá, modificado y traducido al Español" es una herramienta facilitadora de uso sencillo y útil para orientarnos en la valoración funcional de un trabajador sensible para un puesto concreto, así como en la propuesta de adaptación a un puesto compatible.

\section{RECOMENDACIONES}

Las valoraciones médicas para un puesto de trabajo en un trabajador especialmente sensible deberían sustentarse, en la medida de lo posible, en la mayor autonomía, comodidad, productividad y tolerabilidad a largo plazo para minimizar el riesgo de sobrecarga que puede aumentar las limitaciones funcionales ya existentes.

Aplicación de un instrumento de estimación de capacidades funcionales en una trabajadora con obesidad mórbida Caridad León Robles, Esperanza Álvarez Maeztu, Mercedes Petralanda Aburto, Isabel Larruscain Garay, Izaskun Sáez Larrucea 


\section{REFERENCIAS BIBLIOGRÁFICAS}

1. Valle M, Otero C. Guía práctica de salud laboral para la valoración de aptitud en trabajadores con riesgo de exposición a carga física. Escuela Nacional de Medicina del Trabajo (ENMT). Instituto de Salud Carlos III. Ministerio de Economía, Competitividad y Ciencia. Madrid. 2015.

2. Real Decreto Legislativo $8 / 2015$, de 30 de octubre.

3. INSHT. Guía Técnica para la evaluación y prevención de los riesgos relativos a la manipulación manual de cargas. Instituto Nacional de Seguridad e Higiene en el Trabajo. 2009.

4. Treasury Board of Canada Secretariat - Canada.ca. Functional Abilities Form. [en línea]. [Fecha de consulta: 01 junio 2017]. Disponible en: https://www.canada.ca/fr/secretariat-conseil-tresor/services/ valeurs-ethique/diversite-equite/gestion-incapacite/formulaire-determination-capacites-fonctionnelles. html

5. Capodaglio P. Functional limitations and occupational issues in obesity: a review. Int J Occup Saf Ergon. 2010; 16(4):507-23.

6. Anandacoomarasamy A, et al. The impact of obesity on the musculoskeletal system. Int J Obes. 2008; 32(2):211-22.

7. Gilleard W, Smith T. Effect of obesity on posture and hip joint moments during a standing task, and trunk forward flexion motion. Int J Obes. 2007; 31(2):267-71

8. Kortt M, Baldry J. The association between musculoskeletal disorders and obesity. Australian Health Review. 2002; 25(6) 207-214.

9. Forhan M, Gill S. Obesity, functional mobility and quality of life. Best Practice \& Research Clinical Endocrinology \& Metabolism. 2013; 27 (2), 129-137.

10. Porto HCD, Pechak CM. Biomechanical effects of obesity on balance. International Journal of Exercise Science. 2012;5(4):301-320.

11. Colim, Ana et al. Differences in muscular activity between obese and non-obese workers during manual lifting. Dyna Rev Fac Nac Minas, Medellín 2016; 83(196), 55-62.

12. Schulte P, Wagner G. Work, Obesity, and Occupational Safety and Health. J Public Health. 2007 March; 97(3): 428-436.

13. P de Lucas et al. Perspectivas En Exploración Funcional Respiratoria: Obesidad y función pulmonar. Arch Bronconeumol. 2004; 40: 27-31.

14. Rabeca C, Ramos P de, Veale D. Complicaciones respiratorias de la obesidad. Arch Bronconeumol. 2011; 47(5):252-261.

15. Gu J, Charles L. Prevalence of work-site injuries and relationship between obesity and injury among U.S. workers: NHIS 2004-2012. J Safety Res. 2016 September; 58: 21-30.

16. ANSES. Évaluation des risques sanitaires liés au travail de nuit. Avis de l'Anses Rapport d'expertise collective. Agence Nationale de Sécurité Sanitaire de l'Alimentation, de l'Environnement et du Travail (ANSES). Rapport, 2016-06, 430 p. Disponible: https://www.anses.fr/en/system/files/AP2011SA0088Ra. pdf [Fecha de consulta: 10 Junio 2017].

17. Instituto Nacional de Seguridad e Higiene en el Trabajo (INSHT). Guía técnica para la evaluación y prevención de los riesgos relativos a la utilización de los lugares de trabajo. INSHT. Madrid. 2015. 


\section{ANEXOS}

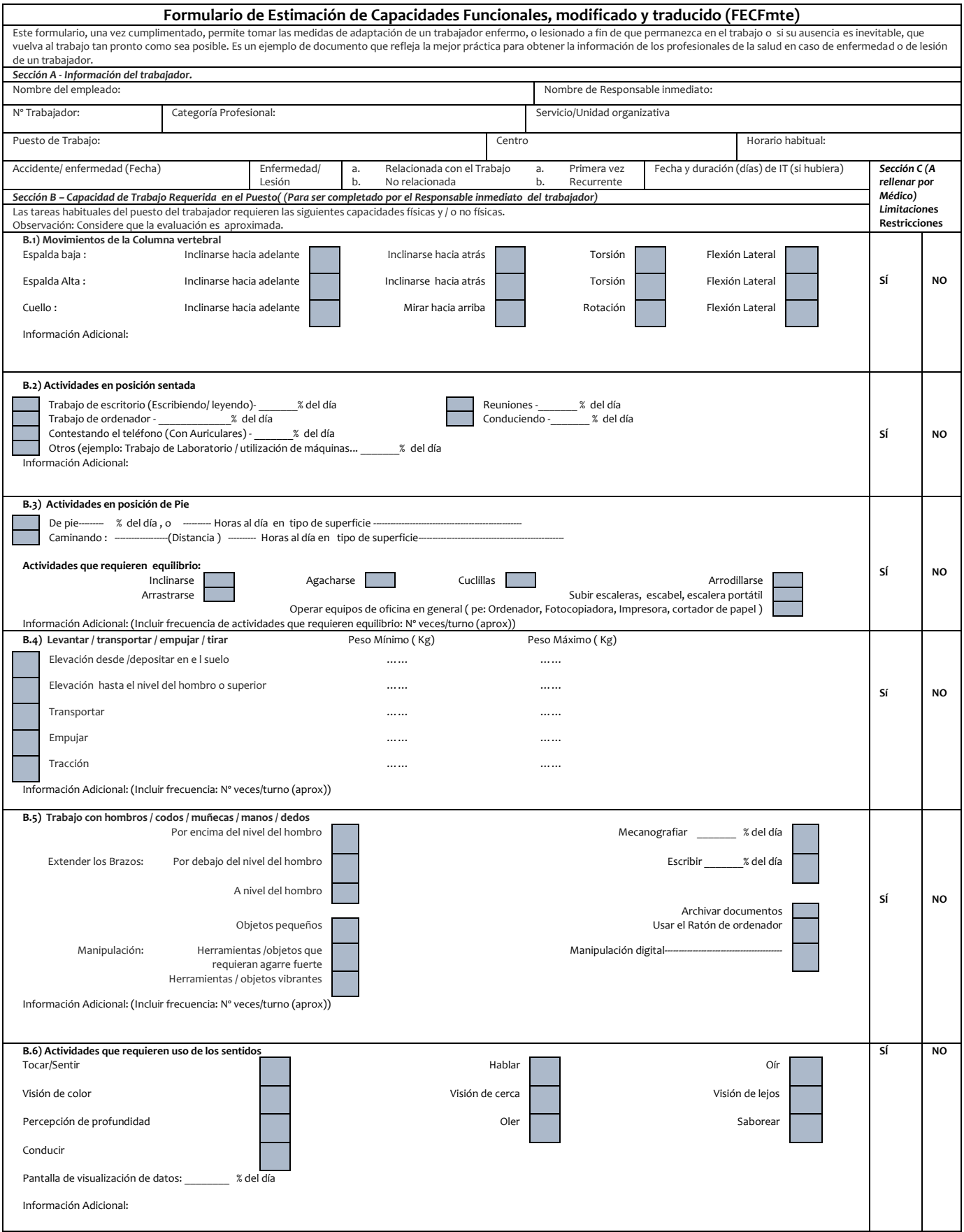




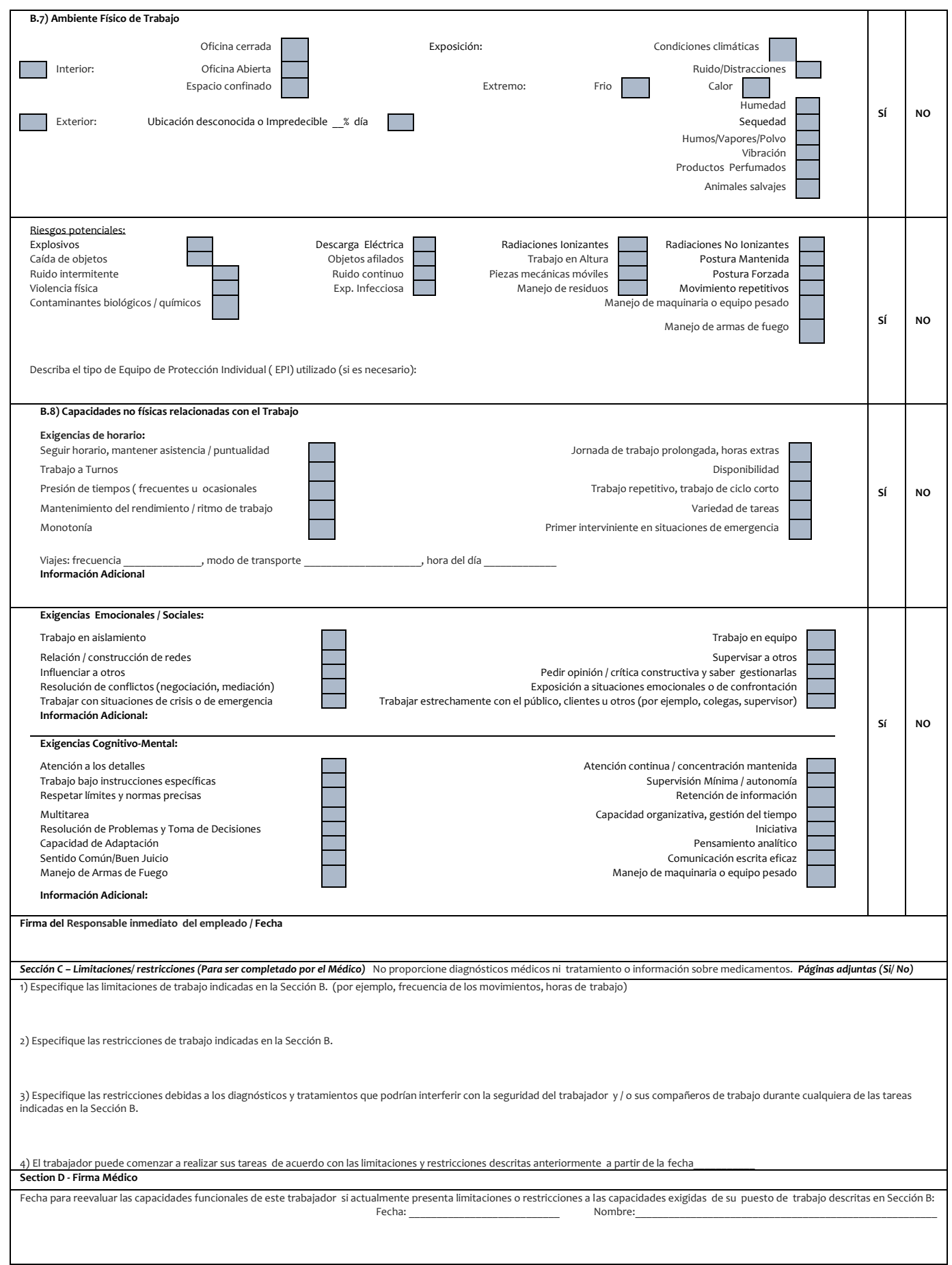

